\title{
Innate Preferences for Radial Gradient Pattern Cues in the Cotton Bollworm, Helicoverpa armigera
}

\author{
Qianwen Luo*, Pu Hu*, Hongfei Zhang, Gaoping Wang, Xianru Guo, Guohui Yuan\#, \\ Weizheng Li" \\ Department of Entomology, College of Plant Protection, Henan Agricultural University, Zhengzhou, China \\ Email: "wei-zhengli@163.com
}

How to cite this paper: Luo, Q.W., Hu, P., Zhang, H.F., Wang, G.P., Guo, X.R., Yuan, G.H. and Li, W.Z. (2018) Innate Preferences for Radial Gradient Pattern Cues in the Cotton Bollworm, Helicoverpa armigera. Advances in Bioscience and Biotechnology, 9, 534-548.

https://doi.org/10.4236/abb.2018.910037

Received: September 19, 2018

Accepted: October 28, 2018

Published: October 31, 2018

Copyright $\odot 2018$ by authors and Scientific Research Publishing Inc. This work is licensed under the Creative Commons Attribution International License (CC BY 4.0).

http://creativecommons.org/licenses/by/4.0/

\begin{abstract}
Information is lacking regarding the visual cues used by Helicoverpa armige$\mathrm{ra}$ moths during nectar feeding. Here, we investigated the preference for radial gradient patterns in $H$. armigera moths. The results indicated that both sexes shared a preference to plain flower models of blue and cyan. The radial gradient pattern (cyan as nectar guide color and blue as petal color) was more attractive than its component plain colors (cyan or blue), especially in male moths. Number of corolla petals did not influence the attractiveness of the cyan-blue pattern. The addition of a tertiary floral attractant to white-blue or cyan-blue radial gradient patterns could dramatically enhance the attractiveness of visual cues in males rather than females, suggesting that males gave a higher weight in olfactory modality than females gave, while females gave a higher weight in vision modality than males gave. All together, we found an optimal combination of floral cues in $H$. armigera sexes as follows: A tertiary floral attractant $(2 \mu \mathrm{L}$ dose of phenylacetaldehyde, benzyl acetate, and salicylaldehyde mixed in 26:15:2) added to white-blue radial gradient flower model ( $3 \mathrm{~cm}$ in diameter). To our knowledge, this is the first time that rose curve and radial gradient tools were used to simulate floral pattern in the studies of flower-visiting insects.
\end{abstract}

\section{Keywords}

Helicoverpa armigera, Flower-Visitation, Pattern, Radial Gradient, Nectar Guide

\section{Introduction}

Studies on floral cues used by Lepidopteron adults during flower visitation have drawn a conclusion: Nocturnal species give higher weight to olfactory cues while

*These authors contributed equally to this work. 
diurnal species give higher weight to visual cues [1]. However, some nocturnal species such as wild Manduca sexta moths did not feed from cloth-bagged fragrant Datura wrightii flowers lacking strong visual contrast, nor did they feed from paper model flowers lacking plant odors, suggesting a synergistic effect between visual and olfactory cues in nectar feeding [2]. Similarly, approaches to odor sources of $M$. sexta naïve males never resulted in proboscis extension or probing in the absence of a visual target [3]. Further studies demonstrated that $M$. sexta flower visitation is based not only on olfactory and visual stimulation per se but also on the temporal and spatial pattern in which these stimuli are perceived, and most responsive was found when both cues were present and emanated from the same source [4]. Additionally, corolla pattern or size could also affect flower visitation. A general knowledge is that the more the patterns like natural flowers, the more they attractive to flower-visiting insects [5] [6] [7].

The cotton bollworm (CBW), Helicoverpa armigera (Hübner), is one of the most serious pest species in north-China. Previously, we have developed an agaropectin formulation of a generic floral attractant [8]. However, information is lacking regarding visual cues used by this agriculturally important noctuid moth during nectar feeding. Since that the synergy between visual cues and olfactory cues also occurred in nocturnal moth species such as $M$. sexta, we believed that a deeper understanding of these factors may be a starting point for the development of highly effective trapping technology system. The patterns of natural flowers could be represented using advanced mathematical tools, such as n-petaled rose curve equations and radial gradient tool [9]. Here, we investigated the preference for radial gradient patterns in CBW moths during dusk foraging. Specifically, we tested the following factors: 1) plain color; 2) radial gradient patterns consisted of two preferable colors; 3) corolla petal number; 4) different nectar guide colors (matched with blue petal); and 5) the combination of floral attractant and preferable patterns.

\section{Materials and Methods}

\subsection{Insects}

Mature larvae were collected in a tobacco field in Scientific \& Educational Precinct, Henan Agricultural University. Helicoverpa armigera and its sibling $H$. assulta often co-occurred in tobacco fields, to a lesser extent, in hot pepper fields. They were identified according to their morphological traits using a microscope [10]. In the laboratory, the larvae were maintained individually in glass tubes and fed with artificial diet under the conditions of $26^{\circ} \mathrm{C} \pm 2^{\circ} \mathrm{C}$ temperature, and $60 \%-70 \%$ relative humidity, 16L:8D photoperiod, with the dark period between 2300 and 0700 hours. Emerged moths were held in cages with cotton gauze as egg-depositing substrate and supplied with $10 \%$ sucrose solution. Test moths were starved for $48 \mathrm{~h}$ to be ready for the bioassays.

\subsection{Chemicals}

Benzyl acetate (99\% purity) and salicylaldehyde (95\% purity) were purchased 
from Sinopharm Chemical Reagent Group Co. Ltd. (Shanghai, China), and phenylacetaldehyde (98\% purity) from Sigma-Aldrich.

\subsection{Preparing of Floral Models}

We used n-petal Rose curves to simulate the visual cues of floral models. These curves can be expressed by a polar equation:

$$
\rho=\cos (n \theta)
$$

Alternately, they can also be expressed by a pair of Cartesian parametric equations:

$$
\begin{aligned}
& x=\cos (n \theta) \times \sin (\theta) \\
& y=\cos (n \theta) \times \cos (\theta)
\end{aligned}
$$

If $\mathrm{n}$ is an integer, the curve will be rose-shaped with $2 n$ petals if $\mathrm{n}$ is even, and $\mathrm{n}$ petals if $\mathrm{n}$ is odd [9].

Drawing of floral models was performed on Microsoft Excel 2003 combined with Adobe Photoshop CS 5. The procedure was as follows: 1) created an arithmetic progression of $\theta$ (initial term $=0.01$, common difference $=0.01$, and total term number $=800$ ) in Microsoft Excel 2003; 2) calculated the corresponding $\mathrm{x}$ and $y$ values based on the above-mentioned Cartesian parametric equations; 3 ) plotted an X-Y scatter graph with smooth curve and without data markers or gridlines; 4) pasted the scatter graph on a newly-created canvas in Photoshop CS 5; 5) input R, G, and B values (see below) of foreground- and background-colors, and completed all the patterns using the combination of paint bucket and radial gradient tools; 6) printed flower models on Ilford Galerie photo paper using a Ricoh Aficio MPC 5501 inkjet color printer. The radial gradient patterns were consisted of two colors, the one as nectar guide color and the other as petal color. For simplicity, we named these patterns from nectar guide color to petal color (e.g., cyan-blue). We think that radial gradient patterns can simulate natural flowers better than those used previously, such as one color cross on another (2 orthogonal lines intersecting at the center) [11]. Some radial gradient pattern examples with four corolla petals are shown in Figure 1.

\subsection{Bioassays}

Bioassays were conducted from 1800 hours to 2200 hours in each evening. Test floral models were arranged at an equal interval in the periphery of the cylinder bottom. A $200 \mu \mathrm{L}$ centrifugal tube contained $100 \mu \mathrm{L} 5 \%$ sucrose solution was fully inserted into the floral foam after passing through the center of each floral model ( $3 \mathrm{~cm}$ in diameter). In each test, 3 - to 4 -day moths starved for $48 \mathrm{~h}$ were introduced in a group of ten individuals via a $2 \mathrm{~cm}$ ID hole in the top cover of a Perspex cylinder ( $50 \mathrm{~cm}$ height $\times 40 \mathrm{~cm}$ ID) placed on a $50 \mathrm{~cm} \times 50 \mathrm{~cm} \times 3 \mathrm{~cm}$ green floral foam. Observation was lasted for $20 \mathrm{~min}$ under a dim illumination provided by white light-emitting diodes. Males and females were tested 


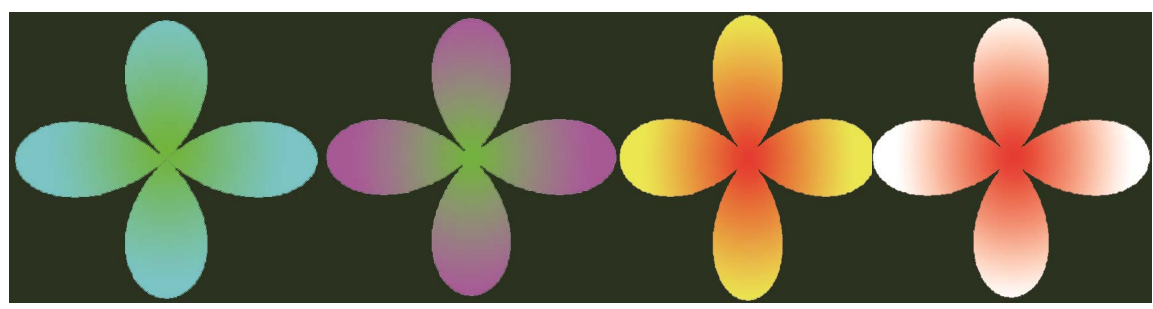

Figure 1. Examples of radial gradient patterns. From left to right: yellow-white, yellow-magenta, red-yellow, and red-white.

separately in all the following experiments. Only probing the sucrose reservoir with the proboscis was counted as a choice.

\subsection{Preference for Plain Color Patterns}

We believe that the two color components of a preferable radial gradient pattern will also be attractive per se. Thus, we firstly tested preference of CBW moths to eight plain-colored flower models to select suitable colors to prepare radial gradient patterns. Eight colors were chosen to test color preference of virgin male and female moths. The RGB values of these colors were as follows: red $(R=255$, $G=B=0)$, green $(R=B=0, G=255)$, blue $(R=G=0, B=255)$, yellow $(R=G$ $=255, \mathrm{~B}=0)$, cyan $(\mathrm{R}=0, \mathrm{G}=\mathrm{B}=255)$, magenta $(\mathrm{R}=\mathrm{G}=255, \mathrm{~B}=0)$, black $(\mathrm{R}$ $=\mathrm{G}=\mathrm{B}=0$ ), and white $(\mathrm{R}=\mathrm{G}=\mathrm{B}=255)$. Reflectance curves (Figure 2) of these colors were determined by an Avantes AvaSpec-ULS3648 fiber optic spectrometer. The positions of eight floral models on the bottom of the cylinder were randomly arranged. A total of 250 males and 250 males were tested.

\subsection{Radial Gradient Patterns}

Based on plain color preference, the preference of CBW moths was tested when presented with a choice among four types of radial gradient patterns (cyan-blue, blue-cyan, blue-green, and green-blue, all $3 \mathrm{~cm}$ in diameter). Each type used two models, and the two models of the same type were placed along a diameter of the cylinder bottom. Positions of different types of models were re-randomized in each test. This experiment was replicated 102 individuals for males and 100 individuals for females.

A second experiment was conducted to determine the necessity of radial gradient combination. The radial gradient pattern mostly preferred by both sexes (cyan-blue) was further tested against each of its two color components (cyan-blue versus cyan, cyan-blue versus blue). A total of eight models, four for each type of pattern, were alternatively arranged. This experiment was replicated 100 individuals for each sex and each treatment.

A third experiment was conducted to determine the effect of corolla petal numbers of the cyan-blue radial gradient pattern on the preference of CBW moths. The corolla was designed as 4,8 , and 12 petals. A total of six models, two for each type of pattern, were randomly arranged. This experiment was replicated 103 individuals for males and 107 individuals for females. 


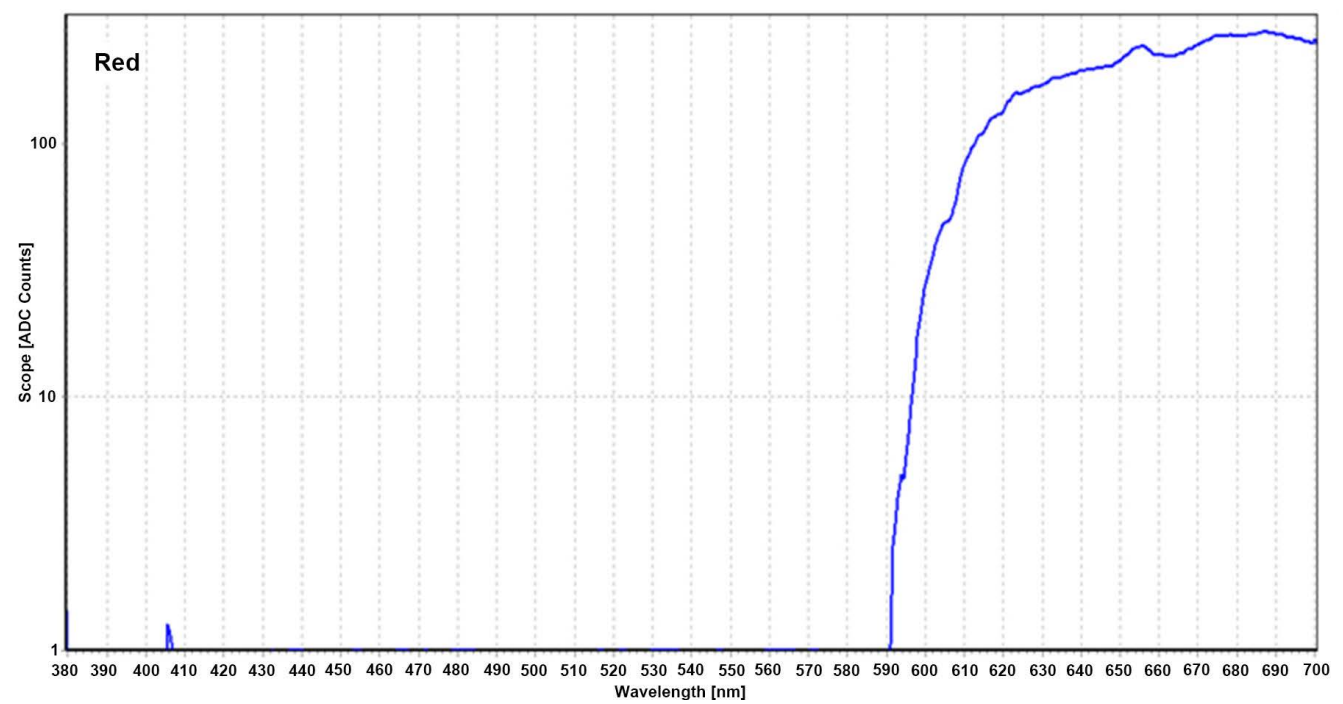

(a)

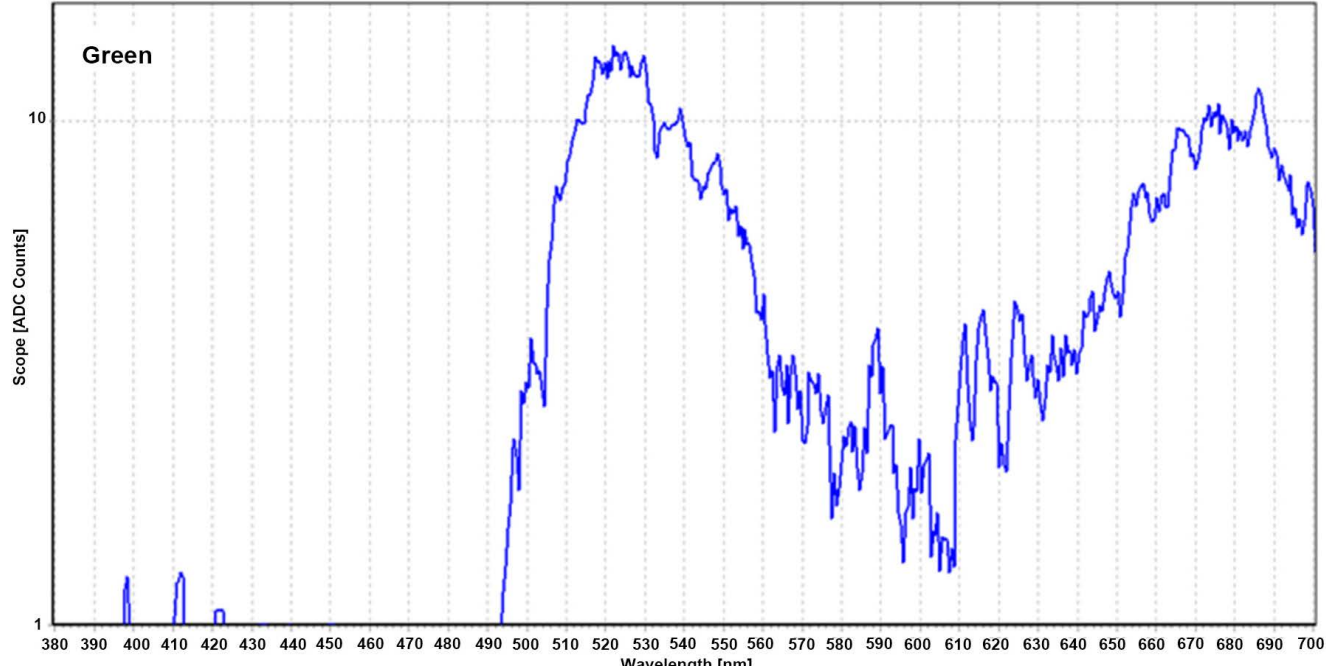

(b)

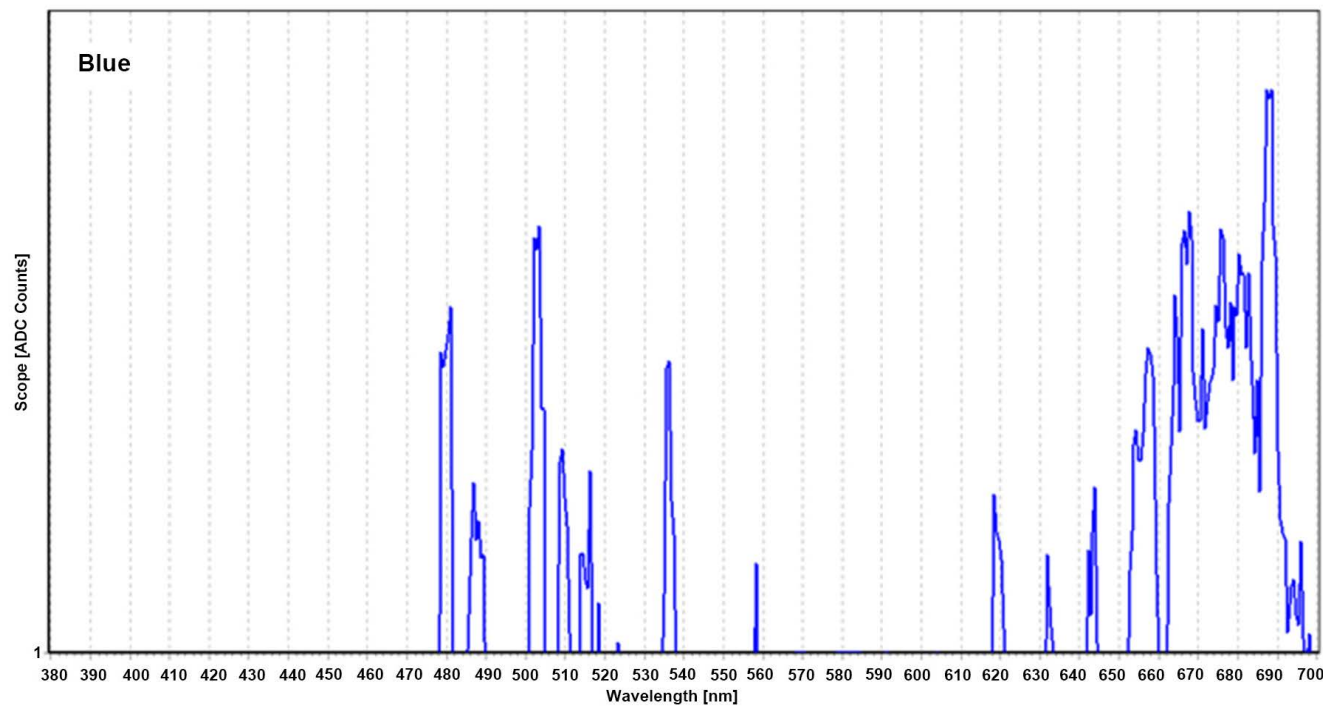

(c) 


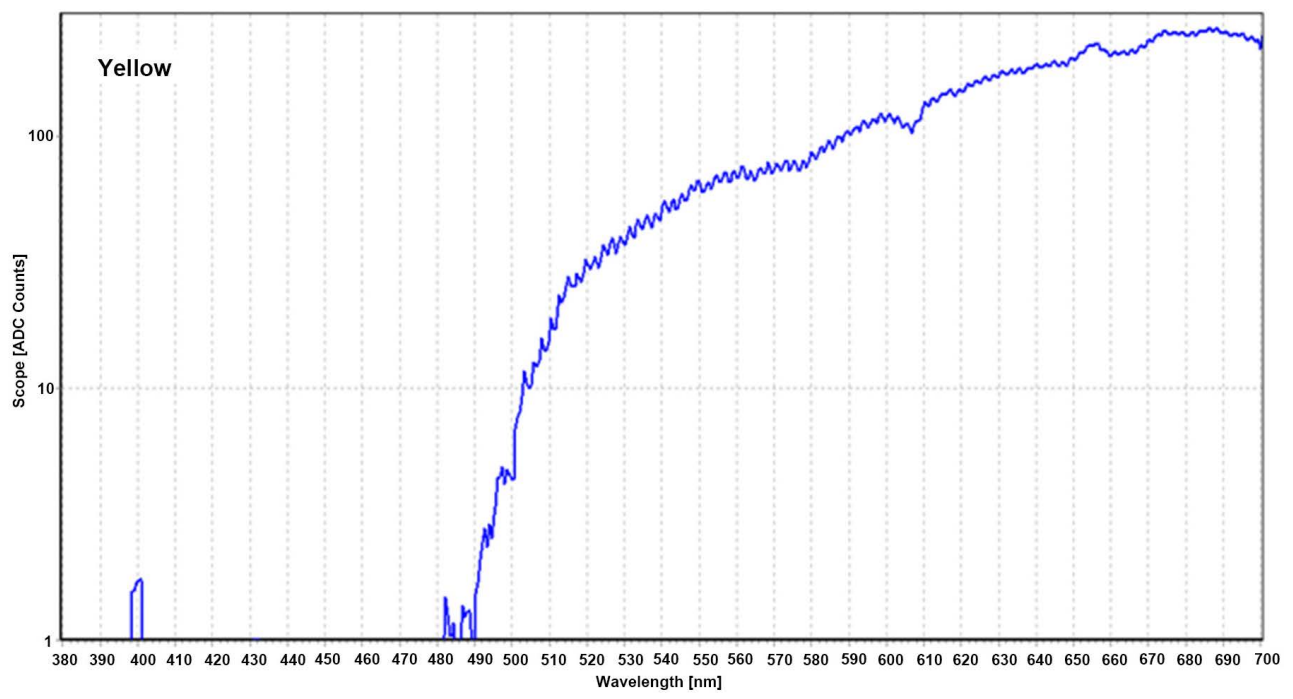

(d)

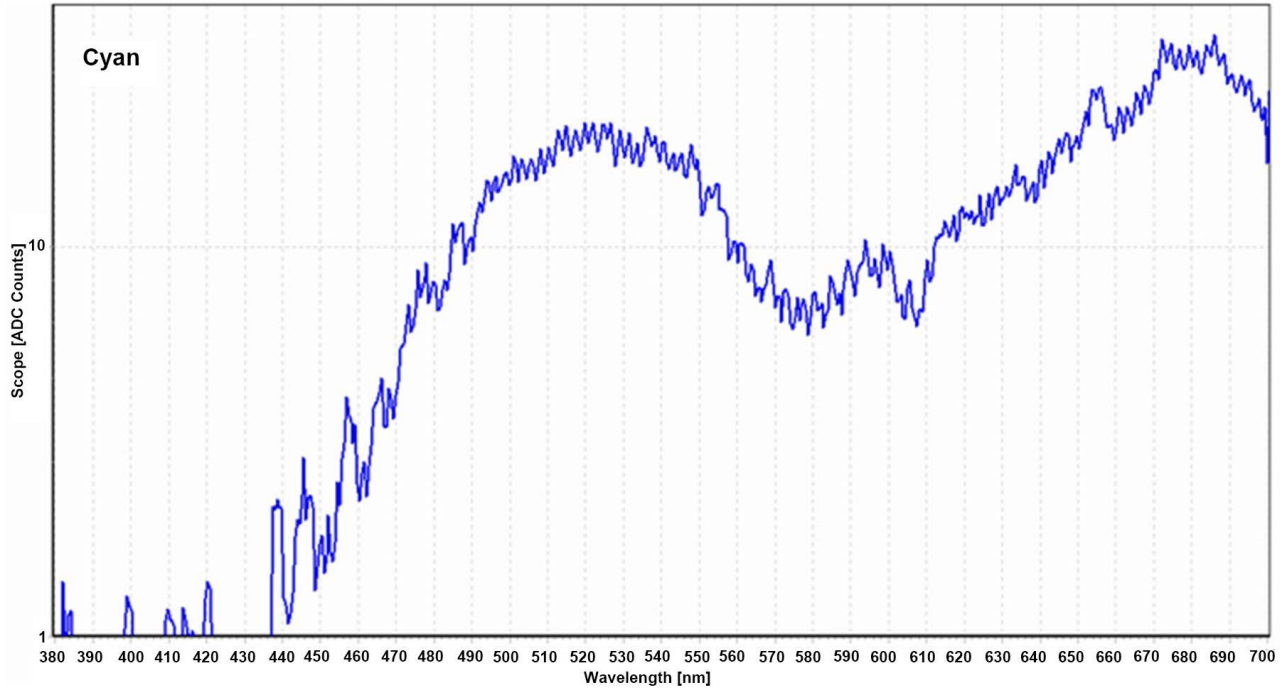

(e)

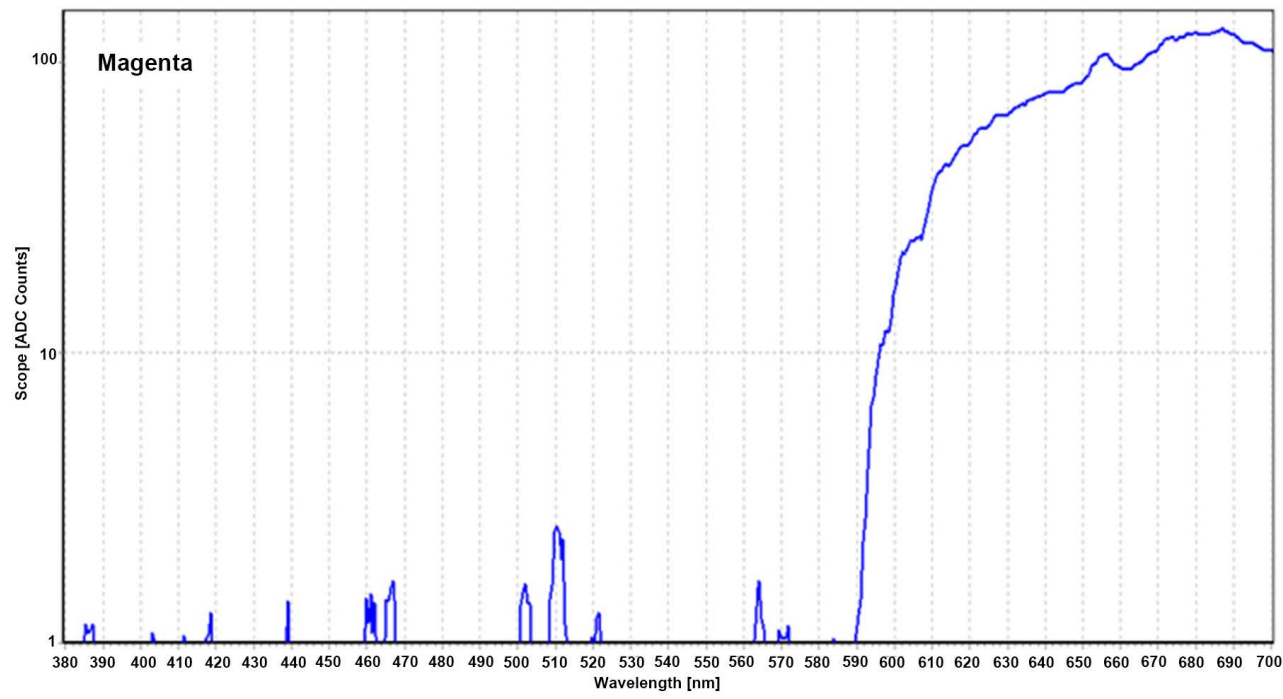

(f) 


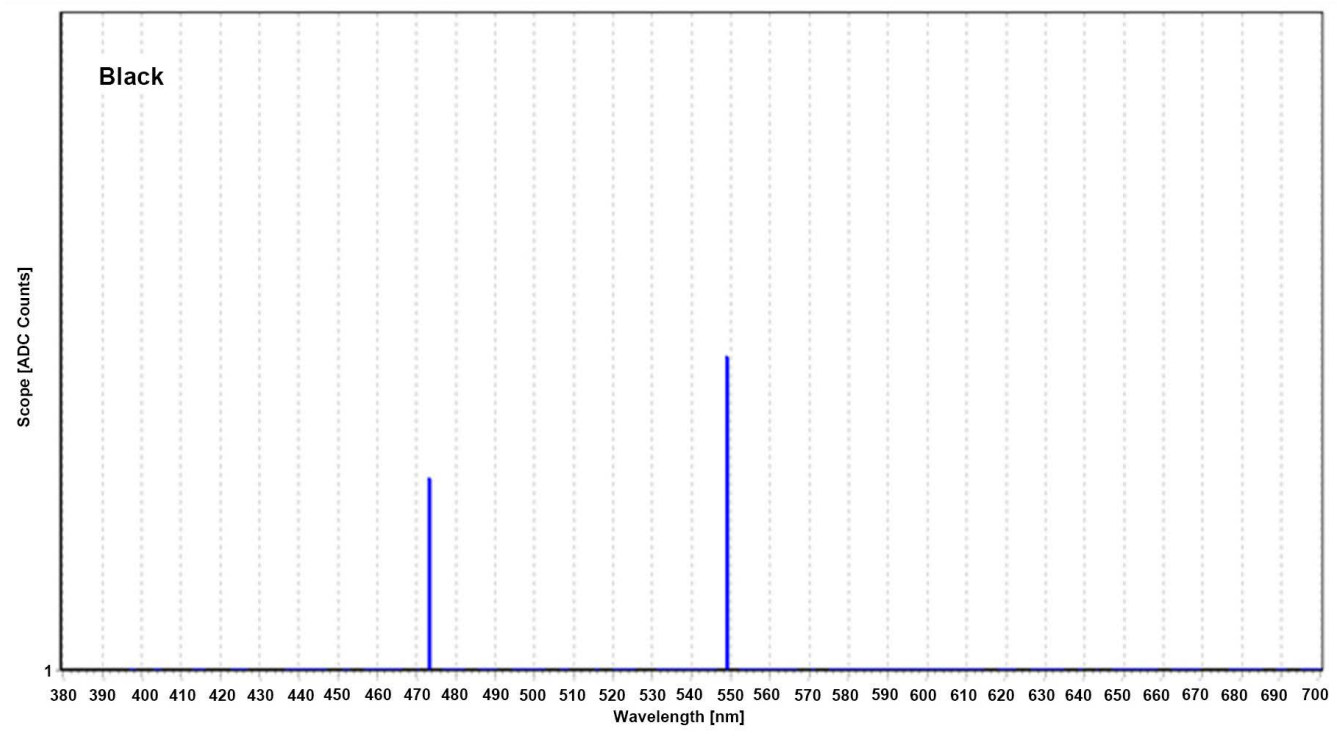

(g)

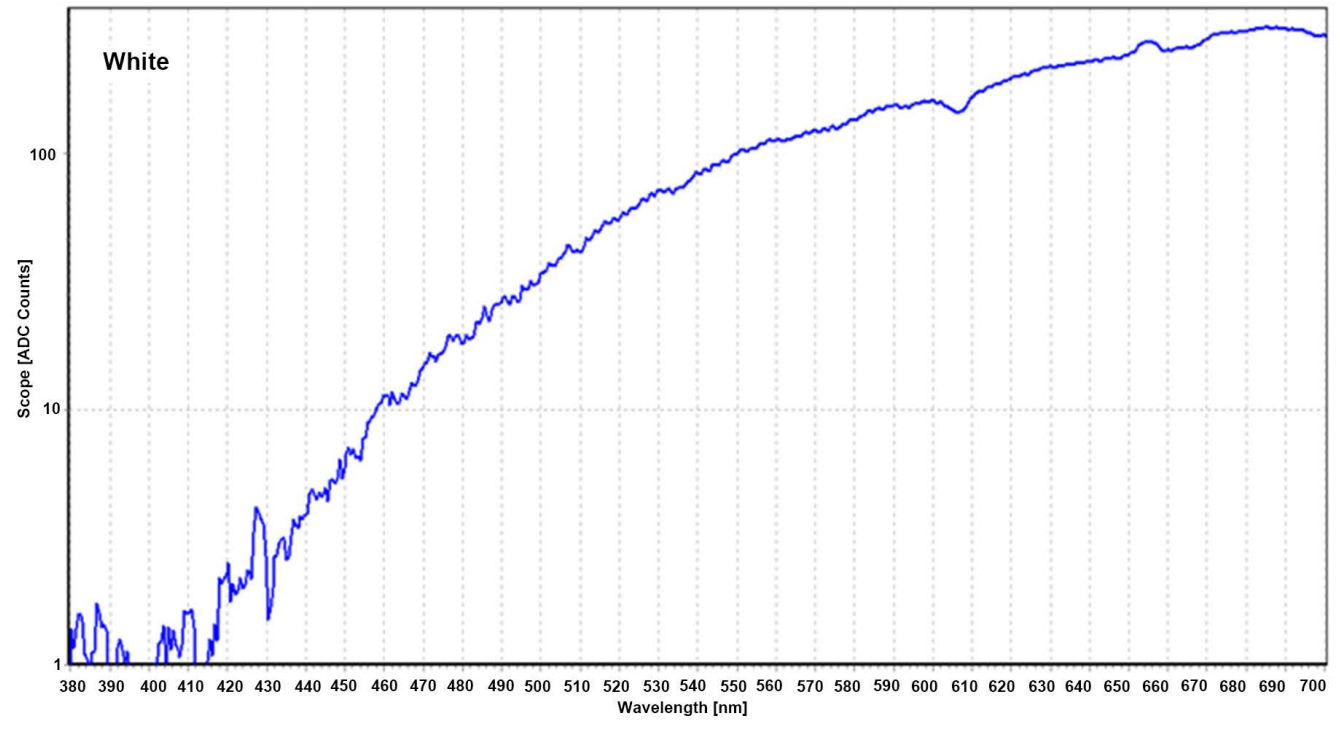

(h)

Figure 2. Reflectance of floral model colors.

A fourth experiment was conducted to determine the effect of four common nectar guide colors (yellow, white, magenta, and cyan) on the preference of CBW moths when combined with blue petal in a radial gradient mode. A total of eight models, two for each type of radial gradient pattern (yellow-blue, white-blue, magenta-blue, and cyan-blue), were randomly arranged in the bioassay apparatus. This experiment was replicated 240 individuals for both sexes.

\subsection{Interaction between Visual Cue and Olfactory Cue}

We compared the preference of CBW moths to different combinations of floral visual cue and floral olfactory cue. A tertiary floral attractant blend $(520 \mu \mathrm{L}$ phenylacetaldehyde plus $300 \mu \mathrm{L}$ benzyl acetate plus $40 \mu \mathrm{L}$ salicylaldehyde) opti- 
mized from field-trapping of noctuid moths [8] was used to provide a generic floral olfactory cue. The tertiary blend was added to $7740 \mu \mathrm{L}$ paraffin oil. This solution $(20 \mu \mathrm{L})$, along with $100 \mu \mathrm{L} 5 \%$ sucrose solution, was added in a $200 \mu \mathrm{L}$ centrifugal tube. Where olfactory cue should be absent, only $100 \mu \mathrm{L} 5 \%$ sucrose solution was added to the centrifugal tube. Four treatments were compared: 1) cyan-blue radial gradient pattern plus olfactory cues; 2) cyan-blue radial gradient pattern; 3) white-blue radial gradient pattern plus olfactory cues; and 4) white-blue radial gradient pattern. The treatments were crossly placed in the periphery of the cylinder bottom. Positions of different treatments were re-randomized in each test. This experiment replicated 100 individuals for both sexes.

\subsection{Statistics}

All the choice frequency data were performed on SPSS for Windows (version: 19.0) and tested by G-test (or likelihood ratio test). The level of significance was set at $\alpha<0.05$.

\section{Results}

\subsection{Preference among 8 Colored Flower Models}

The distribution of colors chosen by naive males of CBW differed significantly from random (Figure 3: $G_{\text {male }}=53.33, d f=7, P<0.0001$ ), while that of those chosen by females did not differ significantly from random $\left(G_{\text {female }}=53.33, d f=\right.$ $7, P=0.0811)$. Significant inter-sex difference was found $\left(G_{7}=43.56, d f=7, P<\right.$ $0.0001)$. Of the eight colors of flower models offered, males showed the strongest preference for cyan and magenta followed by blue, while naïve females showed the strongest preference for blue and green, followed by cyan.

\subsection{Radial Gradient Patterns}

Among the four types of radial gradient patterns (blue-cyan, cyan-blue, blue-green, and green-blue), both sexes of CBW showed the strongest preference for the pattern consisted of cyan nectar guide combined blue petal. The distribution of models chosen by males did not differ significantly from random (Figure 4: $\left.G_{\text {male }}=4.61, d f=3, P=0.2030\right)$, while those chosen by females differed significantly from random $\left(G_{\text {female }}=13.04, d f=3, P=0.0045\right)$. However, there was no significant difference between the choice response exhibited by male and female moths $\left(G_{3}=3.16, d f=3, P=0.3681\right)$.

The radial gradient pattern mostly preferred by two sexes (cyan-blue) was further tested against its two color components (Figure 5). Male moths showed no significant difference of the choice frequencies between the radial gradient model and its two component colors ( $G_{\text {cyan-blue vs. blue }}=2.57, d f=1, P=0.1088$; $\left.G_{\text {cyan-blue vs. cyan }}=0.04, d f=1, P=0.8415\right)$. Female moths also showed no significant difference of the choice frequencies between the radial gradient model and one of its component color, cyan ( $G=1.44, d f=1, P=0.2296)$. In contrast, there 


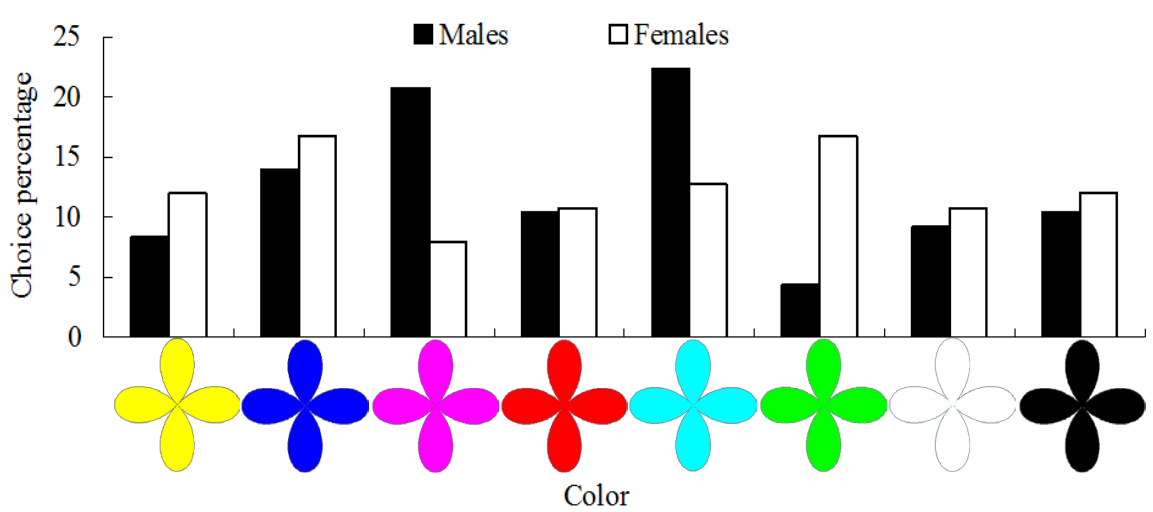

Figure 3. Innate color choices of $H$. armigera moths on colored flower models. The models were made of saturated red, yellow, green, cyan, blue, magenta, black, and white printing papers. All the models were placed on the edge of a floral foam plate. The signs of significances were omitted to simplify the graph, please refer to the text.

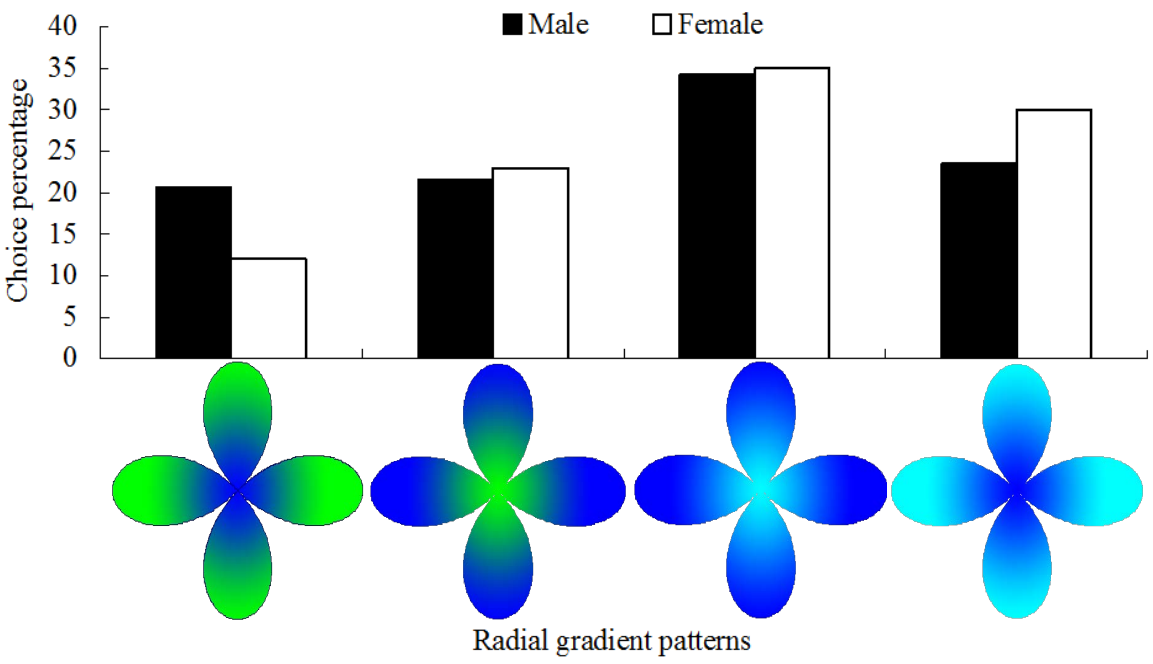

Figure 4. Color choice responses of $H$. armigera male and female moths on radial gradient flower models. Four radial gradient patterns were tested: blue nectar guide plus green petal, green nectar guide plus blue petal, cyan nectar guide plus blue petal, and blue nectar guide plus cyan petal. The signs of significances were omitted to simplify the graph, please refer to the text.

was significant difference of the choice frequencies of the females between the radial gradient model and blue ( $G=6.84, d f=1, P=0.0089$ ). Males and females showed no significant difference between their choice responses $(G=1.02, d f=$ $1, P=0.3124)$.

\subsection{Corolla Petal Numbers}

Both males and females showed no significant difference among the radial gradient patterns with 4, 8, and 12 corolla petals (Figure 6: $G_{\text {male }}=4.08, d f=2, P=$ $\left.0.1301 ; \mathrm{G}_{\text {female }}=1.51, d f=2, P=0.4705\right)$. Between-sex difference was also not significant $(G=4.98, d f=2, P=0.0828)$. A slight bias to 4 corolla petal was found when the data from two sexes were pooled. 


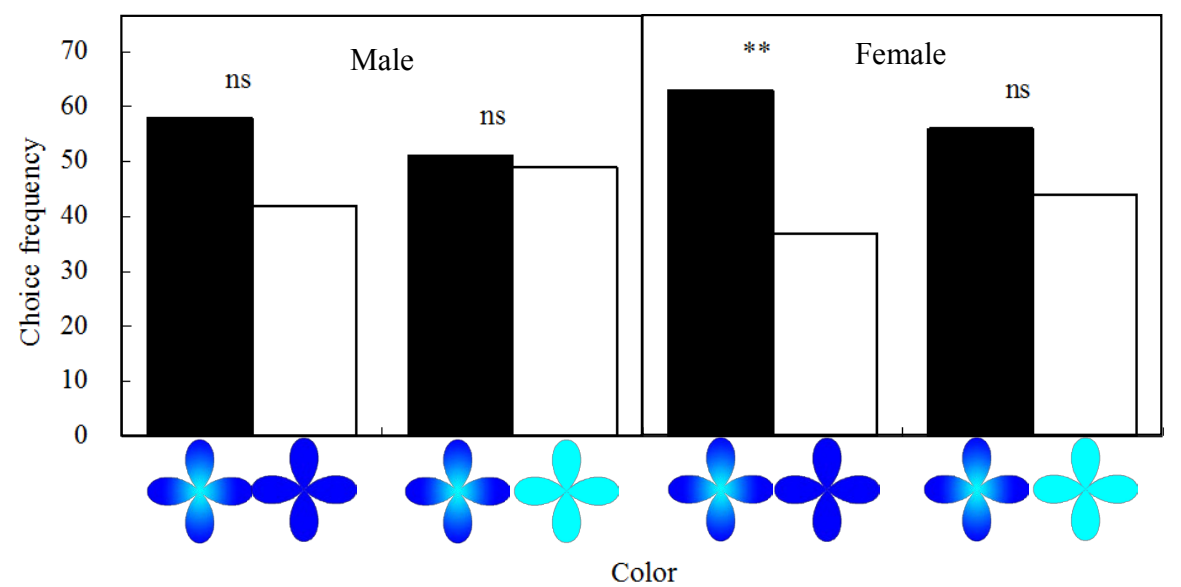

Figure 5. Choice responses of $H$. armigera male and female moths on radial gradient flower model (cyan nectar guide plus blue petal) against its two color components. " $\star \star$ " and "ns" attached above the bar pairs indicate significant and no significant within-group difference at $P=0.05$ level, respectively, tested by Yate's corrected chi-square test.

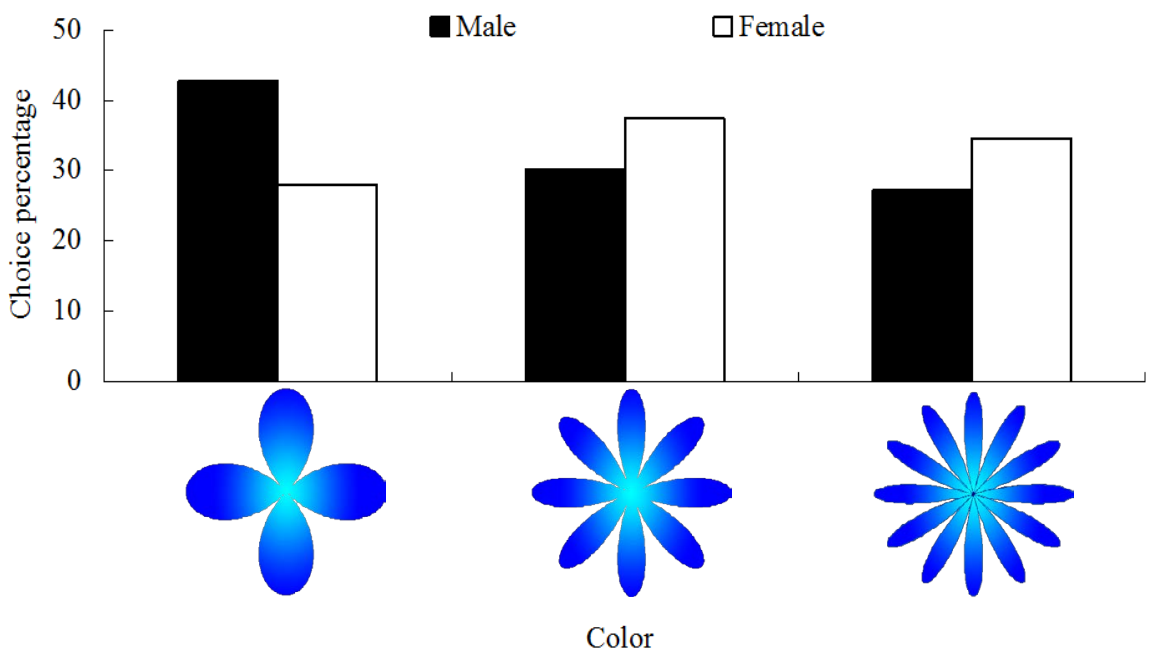

Figure 6. Choice response of male and female $H$. armigera moths to different corolla lobes of the radial gradient pattern (cyan nectar guide plus blue petal). No significant difference was detected in the choice frequencies of different patterns within male moths, within female moths, or between the two sexes.

\subsection{Nectar Guide Colors}

Male CBW moths showed significant difference of the choice frequencies among different nectar guide colors combined with blue petal $(G=106.16, d f=3, P<$ 0.0001 ), so did the females ( $G=16.23, d f=3, P=0.0010$ ). There was significant difference between the choice response presented by male and female moths ( $G$ $=36.38, d f=3, P<0.0001)$, and this statistical result was caused by the strong preference of males to the cyan nectar guide color plus blue petal (Figure 7).

\subsection{Interaction between Floral Olfactory Cues and Visual Cues}

On a whole, choice frequencies of the four treatments chosen by CBW males 


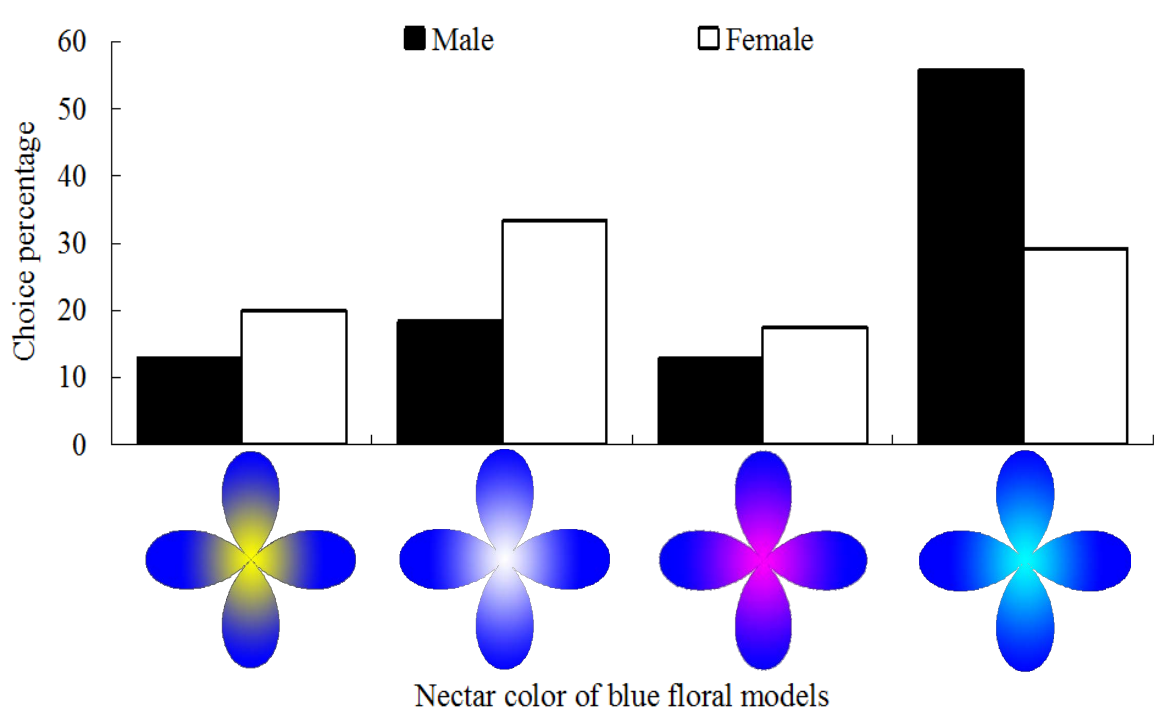

Figure 7. Choice response of male and female $H$. armigera moths to different nectar guide color of the radial gradient pattern (petal color: blue). The signs of significance were omited to simplify the graph, please refer to the text.

showed significant difference ( $G=25.45, d f=3, P<0.0001$ ), while choice frequencies of the four treatments chosen by females did not show significant difference ( $G=7.32, d f=3, P=0.0623)$. When the data from the four treatments were pooled according to different comparison views, the choice frequencies of males to the two olfactory cues-present patterns were significantly higher than the two olfactory cues-absent patterns $(G=16.46, d f=1, P<0.0001)$. In contrast, when the data from the same visual cue were pooled together, no significant difference was observed between different visual cues $(G=2.57, d f=1, P=$ 0.1088 ). Contrary to the statistical results from the males, choice frequencies of females to the two olfactory cue-present treatments and the two olfactory cue-absent treatments did not show significant difference $(G=1.97, d f=1, P=$ 0.1608). In contrast, they preferred the two white-blue patterns to the two cyan-blue patterns ( $G=4.03, d f=1, P=0.0448)$ (Figure 8).

\section{Discussion}

To the best of our knowledge, this is the first time that Rose Curve and radial gradient were used to simulate floral pattern in insect preference studies.

\subsection{Plain Colored Models}

We found that both sexes of CBW moths prefer to blue and cyan in the bioassay of plain colored models, but males showed stronger preference to magenta than females, and females showed stronger preference to green than males. An explanation to the inter-sexual differentiation to green might be that female insects had the motivation of laying eggs on green leaves [12]. We expected that males should also exhibit strong preference to green, since it is one of the three primary colors in most arthropods [13], and finding a green plant is the first step to 


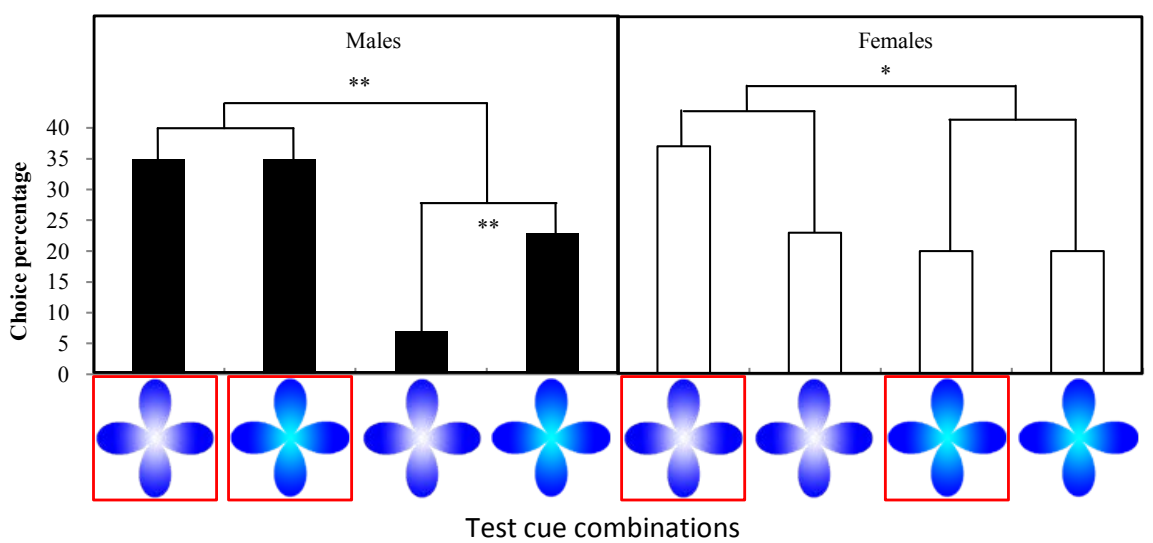

Figure 8. Choice response of $H$. armigera male and female moths to different combinations of floral visual cues and floral olfactory cues. In the testing arena, four floral models were presented together. Among them, two patterns were white neactar guide combined with blue petals, the other two patterns were cyan neactar guide combined with blue petals. Different visual cues were arranged alternately. Patterns with red frames indicate the addition of floral olfactory cues. " $* *$ ” and "*” attached above the bar pairs indicate significant difference at $P=0.01$ and $P=0.05$ levels, tested by Yate's corrected chi-square test. To simplify the graphs, the signs of the overall significances as well as the comparison items without significance were omited, please refer to the text.

forage on its flower. However, visiting preference of CBW males to green is not the fact in this bioassay. This may be due to the puzzling effect of the combination of a flower-like shape combined with a nonflower-like color on these males.

Innate bias of CBW moths to blue was similar to another nocturnal moth species, M. sexta [14] [15]. Blue flowers have one property that makes them a good choice for anthophilous insects: Most natural blue objects are flowers with the exception of sky, providing color contrast with the background [14] [15].

Surprisingly, both sexes of CBW moths exhibited a fairly low response to red, yellow, white, and black colors even most of these colors were more common in natural flowers than blue and green colors. We believe that, just like M. sexta [14] [15], innate preference and learnt preference to flower colors in CBW moths are very different. Alternatively, an interesting explanation is that red, yellow, white, and black colors are the most common warning coloration in natural enemies such as predatory wasps and ladybirds.

\subsection{Radial Gradient Patterns}

Visiting frequencies of CBW moths to the radial gradient patterns consisted of the shared preferable color (blue and cyan) were higher than those consisted of blue and green (males: $57.84 \%$ vs. $42.16 \%$; females: $65.00 \%$ vs. $35.00 \%$ ). Additionally, within-sex analyses indicated that the discriminating ability of females was slightly stronger than that of males. Cyan-blue radial gradient pattern was stronger than its color components. Again, the preference of females to radial gradient pattern against its color components was slightly stronger than that of males. Comparison of different nectar guide colors matched with blue petals in- 
dicated that both sexes showed fairly strong discriminating ability. White-blue and cyan-blue radial gradient patterns elicited stronger preference of females than the other two patterns. Preference for cyan-blue radial gradient pattern in males was much stronger than the other three patterns, and this may be due to the strong preference of males to its two color components. Surprisingly, plain magenta color could also elicit strong response of males, but when it was used as nectar guide color and matched with blue petal, the pattern elicit very low response of males. Whether the radial gradient patterns consisted of the two strongest plain colors (cyan and magenta) could show stronger response in males than the cyan-blue pattern need to be explored in the future. Both sexes showed fairly weak discriminating ability to the number of corolla petals.

\subsection{Interactions between Olfactory Cues and Visual Cues}

The addition of floral olfactory cues in floral visual cues could dramatically enhance the attractiveness of radial gradient patterns, and this effect was more salient in the white-blue pattern than in the cyan-blue pattern. The addition of floral olfactory cues could override the specific preference of males to cyan-blue pattern, but the enhancement effect of floral olfactory cues could only occur in the white-blue pattern rather than the cyan-blue pattern. This suggested that males gave a higher weight in olfactory modality than females gave, while females gave a higher weight in vision modality than males gave.

All together, through artificial simulation method, we found the optimal combination of floral cues in CBW moths as follows: a tertiary floral attractant ( $2 \mu \mathrm{L}$ dose of phenylacetaldehyde, benzyl acetate, and salicylaldehyde mixed in 26:15:2) added to white-blue radial gradient flower model ( $3 \mathrm{~cm}$ in diameter).

\subsection{Future Implications}

Some additional flower features also affect moth foraging on flowers, such as corolla surface area, floral tactile cues, floral humidity gradient, and carbon dioxide emitted from opening flowers. Kelber [5] found that flower models of approximately $32 \mathrm{~mm}$ in diameter (similar to our study) were preferred to smaller and larger ones in M. stellatarum. Similarly, Goyret \& Raguso [16] also reported that corolla surface area negatively affects flower handling efficiency of M. sexta. The nationwide collection of field data suggested that the sizes of flowers utilized by butterflies were relevant to the body sizes of these butterflies [17]. Therefore, we think it appropriate to use smaller flower models to test preference of CBW moths on the ground that the body sizes of the two hawkmoth species are much bigger than our subject. Two hawkmoth species, M. stellatarum [11] and M. sexta [18], could improve their flower inspection efficiency when visual floral guides or tactile guides (grooves) presented on tested flower models. Additionally, it has been demonstrated that floral cues of moths could also involve floral humidity [19] or plant primary metabolites such as carbon dioxide [14] [15] [20]. All of these factors will be investigated in the near future. 


\section{Acknowledgements}

The authors thank Xiaohui Teng, a master student of Henan Agricultural University, for preparing of test flower models, Huiling Li of Institute of Plant Protection, Henan Academy of Agricultural Sciences for measuring color parameters, four undergraduate trainees (Yingying Zheng, Zijing Liu, Yutong Zhuang, and Mingzhu Yuan) for insect rearing and part bioassay works.

\section{Conflicts of Interest}

The authors declare no conflicts of interest regarding the publication of this paper.

\section{References}

[1] Balkenius, A., Rosén, W. and Kelber, A. (2006) The Relative Importance of Olfaction and Vision in a Diurnal and a Nocturnal Hawkmoth. Journal of Comparative Physiology A, 192, 431-437. https://doi.org/10.1007/s00359-005-0081-6

[2] Raguso, R.A. and Willis, M.A. (2005) Synergy between Visual and Olfactory Cues in Nectar Feeding by Wild Hawkmoths, Manduca sexta. Animal Behaviour, 69, 407-418. https://doi.org/10.1016/j.anbehav.2004.04.015

[3] Raguso, R.A. and Willis, M.A. (2002) Synergy between Visual and Olfactory Cues in Nectar Feeding by Naive Hawkmoths Manduca sexta. Animal Behaviour, 64, 685-695. https://doi.org/10.1006/anbe.2002.4010

[4] Goyret, J., Markwell, P.M. and Raguso, R.A. (2007) The Effect of Decoupling Olfactory and Visual Stimuli on the Foraging Behavior of Manduca sexta. Journal of Experimental Biology, 210, 1398-1405. https://doi.org/10.1242/jeb.02752

[5] Kelber, A. (1997) Innate Preferences for Flower Features in the Hawkmoth Macroglossum Stellatarum. Journal of Experimental Biology, 200, 827-836. https://www.ncbi.nlm.nih.gov/pubmed/9318606

[6] Kelber, A. (2002) Pattern Discrimination in a Hawkmoth: Innate Preference, Learning Performance and Ecology. Proceedings of the Royal Society B, 269, 2573-2577. https://doi.org/10.1098/rspb.2002.2201

[7] Simonds, V. and Plowright, C.M.S. (2004) How do Bumblebees First Find Flowers? Unlearned Approach Responses and Habituation. Animal Behaviour, 67, 379-386. https://doi.org/10.1016/j.anbehav.2003.03.020

[8] Li, W., Li, H., Wang, J., Guo, X., You, X. and Yuan, G. (2014) Optimization of Floral Attractant Formula with Broad-trapping Spectrum of Moths Using Uniform Design. Chinese Agricultural Science Bulletin, 30, 304-311 (in Chinese with English abstract).

[9] Compiling Group (1979) Mathematical Handbook. 1st Edition, Higher Education Press, Beijing.

[10] Hu, P., Li, H.L., Zhang, H.F., Luo, Q.W., Guo, X.R., Wang, G.P., Li, W.Z. and Yuan, G.H. (2018) Experience-Based Mediation of Feeding and Oviposition Behaviors in the Cotton Bollworm: Helicoverpa armigera (Lepidoptera: Noctuidae). Plos One, 13, e0190401. https://doi.org/10.1371/journal.pone.0190401

[11] Goyret, J. and Kelber, A. (2011) How Does a Diurnal Hawkmoth Find Nectar? Differences in Sensory Control with a Nocturnal Relative. Behavioral Ecology, 22, 976-984. https://doi.org/10.1093/beheco/arr078 
[12] Weiss, M.R. and Papaj, D.R. (2003) Colour Learning in Two Behavioural Contexts: How Much Can a Butterfly Keep in Mind? Animal Behaviour, 65, 425-434. https://doi.org/10.1006/anbe.2003.2084

[13] Briscoe, A.D. and Chittka, L. (2001) The Evolution of Color Vision in Insects. Annual Review of Entomology, 46, 471-510. https://doi.org/10.1146/annurev.ento.46.1.471

[14] Goyret, J., Markwell, P.M. and Raguso, R.A. (2008) Context- and Scale-Dependent Effects of Floral $\mathrm{CO}_{2}$ on Nectar Foraging by Manduca sexta. Proceedings of the National Academy of Sciences, 105, 4565-4570. https://doi.org/10.1073/pnas.0708629105

[15] Goyret, J., Pfaff, M., Raguso, R.A. and Kelber, A. (2008) Why Do Manduca sexta Feed from White Flowers? Innate and Learnt Colour Preference in a Hawkmoth. Naturwissenschaften, 95, 569-576. https://doi.org/10.1007/s00114-008-0350-7

[16] Goyret, J. and Raguso, R.A. (2006) The Role of Mechanosensory Input in Flower Handling Efficiency and Learning by Manduca sexta. Journal of Experimental Biology, 209, 1585-1593. https://doi.org/10.1242/jeb.02169

[17] Kandori, I., Yamaki, T., Okuyama, S., Sakamoto, N. and Yokoi, T. (2009) Interspecific and Intersexual Learning Rate Differences in Four Butterfly Species. Journal of Experimental Biology, 212, 3810-3816. https://doi.org/10.1242/jeb.032870

[18] Goyret, J. (2010) Look and Touch: Multimodal Sensory Control of Flower Inspection Movements in the Nocturnal Hawkmoth Manduca sexta. Journal of Experimental Biology, 213, 3676-3682. https://doi.org/10.1242/jeb.045831

[19] von Arx, M., Goyret, J., Davidowitz, G. and Raguso, R.A. (2012) Floral Humidity as a Reliable Sensory Cue for Profitability Assessment by Nectar-Foraging Hawkmoths. Proceedings of the National Academy of Sciences, 109, 9471-9476. https://doi.org/10.1073/pnas.1121624109

[20] Thöm, C., Guerenstein, P.G., Mechaber, W.L. and Hildebrand, J.G. (2004) Floral $\mathrm{CO}_{2}$ Reveals Flower Profitability to Moths. Journal of Chemical Ecology, 30, 1285-1288. https://doi.org/10.1023/B:JOEC.0000030298.77377.7d 